\title{
Fractional flow reserve to guide surgical coronary revascularization
}

\author{
Tara Shah ${ }^{1}$, Joshua D. Geleris ${ }^{2}$, Ming Zhong ${ }^{1}$, Rajesh V. Swaminathan ${ }^{3}$, Luke K. Kim ${ }^{1}$, Dmitriy N. Feldman ${ }^{1}$ \\ ${ }^{1}$ Division of Cardiology, ${ }^{2}$ Department of Medicine, Weill Cornell Medical College, New York Presbyterian Hospital, New York, NY 10065, USA; \\ ${ }^{3}$ Duke University Medical Center, Duke Clinical Research Institute, Durham, NC 27705, USA \\ Contributions: (I) Conception and design: T Shah, D Feldman; (II) Administrative support: None; (III) Provision of study materials or patients: None; \\ (IV) Collection and assembly of data: T Shah; (V) Data analysis and interpretation: All authors; (VI) Manuscript writing: All authors; (VII) Final \\ approval of manuscript: All authors. \\ Correspondence to: Tara Shah, MD. Division of Cardiology, Weill Cornell Medical College, New York Presbyterian Hospital, 520 East 70 th Street, \\ Starr-434 Pavilion, New York, NY 10021, USA. Email: tas2012@nyp.org.
}

\begin{abstract}
Coronary angiography has traditionally been used as the final diagnostic tool in the evaluation of coronary artery disease (CAD). However, conventional angiography identifies anatomically obstructive coronary disease, but it is limited in its ability to identify hemodynamically significant lesions. The emergence of fractional flow reserve (FFR) technology, in conjunction with angiography, offers a functional, as well as anatomic, assessment of epicardial coronary obstructions. Several pivotal studies have demonstrated that FFR-guided coronary revascularization is a safe and effective in patients with single and multivessel CAD. There are emerging data to suggest that FFR may also play an integral role in planning surgical revascularization and in the evaluation of post-coronary artery bypass patients and their graft patency. This review will explore the physiologic underpinnings of FFR methodology, its clinical value and limitations, and its applications in coronary artery bypass grafting (CABG) surgery.
\end{abstract}

Keywords: Fractional flow reserve (FFR); percutaneous coronary intervention (PCI); coronary artery bypass grafting (CABG)

Submitted Jan 31, 2017. Accepted for publication Mar 01, 2017.

doi: $10.21037 /$ jtd.2017.03.55

View this article at: http://dx.doi.org/10.21037/jtd.2017.03.55

\section{Introduction}

Invasive coronary angiography has long been considered as the gold standard method for the diagnosis of obstructive coronary artery disease (CAD). However, the physiology of coronary arterial blood flow is complex, and there is frequent discordance between the visual assessment of lesions' degree of stenosis and its effect on myocardial perfusion. Fractional flow reserve (FFR) measurement is an invasive means of assessing the physiologic significance of an epicardial coronary artery stenosis. FFR has dramatically changed our understanding of the complex relationship between anatomical stenosis and functional flow limitations. It has become an invaluable tool in clinical practice to guide the decision-making in coronary revascularization and type of coronary revascularization. Recently, instantaneous wave free ratio (iFR) has been introduced in interventional practice, a technology that does not require administration of pharmacologic hyperemia. This article provides a practical overview of the physiologic principles that guide FFR measurement, sheds light on its nuances, important studies investigating its use, and how it can be applied to guide surgical coronary revascularization. FFR may play a unique role in determining the need and best revascularization strategy, planning the extent of the surgical revascularization and evaluating patients with residual coronary disease after coronary artery bypass surgery (CABG). This review will help to understand potential pitfalls and limitations of the FFR measurements in various complex clinical and anatomical situations. 


\section{FFR physiology and measurement}

Understanding the physiology of coronary blood flow is integral to understanding FFR methodology and its limitations. Coronary blood flow is determined by three major components: epicardial artery resistance, arteriolar and microcirculatory resistance and lastly, time-varying compressive resistance that is higher in the subendocardial than subepicardial layers (1). Each of these components can significantly contribute to blood flow disturbances. First, while epicardial resistance is negligible under normal circumstances, with the development of $>50 \%$ luminal narrowing, resistance increases until it reduces resting myocardial flow. The second component of coronary resistance is primarily determined by the size and density of the arterioles, which are modulated by metabolic and autoregulatory changes that can maintain perfusion across a wide range of hemodynamic conditions. The third component varies throughout the cardiac cycle. During systole, cardiac contraction creates backpressure and, thus, a reduction in driving pressure for coronary flow that impedes its ability to perfuse the subendocardium. When left ventricular pressure is elevated (e.g., in leftsided heart failure), this also causes passive compression of microcirculation during diastole (2). When an epicardial coronary stenosis is present, there is an increase in resistance to flow, causing the distal arterioles to dilate in order to maintain basal coronary flow. Although flow may be maintained at resting condition, any state, which increases myocardial oxygen demand, will result in less incremental vasodilatation and a lower coronary flow velocity reserve.

Conceptually, FFR is used to determine the physiologic significance of an epicardial stenosis by measuring the drop in perfusion pressure across coronary narrowing, which is related to the rate of flow. This pressure gradient during maximal hyperemia, as opposed to a resting pressure gradient, is related to the flow limiting potential of a lesion (3). The FFR is commonly expressed as a ratio of the maximum achievable blood flow across a stenosis in the epicardial coronary artery to the maximum achievable blood flow across that artery in the absence of any stenosis. As coronary blood flow is difficult to measure directly, FFR is obtained from a pressure-based measurement, where a pressure sensor tipped coronary wire is used to simultaneously measure the pressure distal to a lesion $\left(\mathrm{P}_{\mathrm{d}}\right)$ and the aortic pressure $\left(\mathrm{P}_{\mathrm{a}}\right)$ proximal to a lesion during induced, maximal coronary hyperemia. These measurements are then used to derive an FFR value based on the mathematical equation FFR $=\left(\mathrm{P}_{d}-\mathrm{P}_{v}\right) /\left(\mathrm{P}_{\mathrm{a}}-\mathrm{P}_{\mathrm{v}}\right)\left[\mathrm{P}_{\mathrm{v}}=\right.$ central venous pressure (CVP), assumed to be negligible] (3). This calculated FFR value is reflective of overall myocardial perfusion through antegrade coronary and collateral flow. However, given that coronary blood flow is not directly measured, the relationship between the pressure gradient and flow is highly dependent on several physiological assumptions that may be inaccurate in certain conditions or cohorts (4). Furthermore, the accuracy of an FFR measurement is contingent on maximizing microvascular vasodilation, as coronary perfusion pressure is assumed to be directly proportional to blood flow during this state. Achieving the state of maximal hyperemia and minimal arteriolar and microcirculatory resistance is thereby very important and is typically done through the administration of intravenous or intracoronary vasodilator agents, such as adenosine (5). Failure to ensure the condition of maximum vasodilation may result in a falsely lower pressure gradient, resulting in a higher calculated FFR value and substantially decreasing the sensitivity (6).

iFR has been recently introduced in clinical practice and is a pressure-based index that does not require administration of pharmacologic hyperemia (7). It is an attractive technology as it may reduce procedural time, lower procedural cost, improve patient comfort and avoid side effects of adenosine, especially for patients at risk. The measurement of iFR is based on several coronary hemodynamic concepts and assumptions (8). First, there is a short period in diastole during which resistance across the coronary vasculature at rest is relatively stable (the "wave free" period). Second, during this time period, intracoronary flow and pressure should be proportional, and a trans-stenotic pressure gradient should approximate flow. Finally, the presence of a pressure gradient at rest is indicative of hemodynamic stenosis severity better than under hyperemic conditions, as the presence of such a gradient indicates evidence of microvascular compensation to preserve flow.

There have been several recent studies that have evaluated the correlation between FFR and iFR measurements. The ADVISE study was the first clinical study that demonstrated a correlation between iFR and FFR measurements ( $r=0.9$, $\mathrm{P}<0.001)$. Using FFR as a reference gold standard with a cutoff of 0.80 , iFR demonstrated a receiver-operating characteristic area under the curve of $93 \%$, suggesting high accuracy (9). The ADVIS E-in-practice (an international multicenter evaluation of iFR in clinical practice) demonstrated that the best cut-off to discriminate lesions 
with FFR $\leq 0.80$ was an iFR value of $\leq 0.9$ (10). Using this cutoff, $21.7 \%$ of lesions had discordant measurements: $16.6 \%$ of lesions with iFR $\geq 0.90$ had FFR $\leq 0.80$ and $5.1 \%$ of lesions with iFR $<0.90$ had FFR $>0.80$. With these thresholds, iFR demonstrated a combination of strong sensitivity ( $81 \%$ ) and specificity (79\%), a negative predictive value of $87 \%$, and a positive predictive value of $71 \%(10)$. Although these data suggest that iFR-based decision making with a cutoff value of 0.90 may defer $16.6 \%$ of "FFR positive" lesions, this value decreases to only $1.3 \%$ when an FFR cutoff of 0.67 is used. In an effort to address some of the discrepancy, the RESOLVE study investigated the correlation between iFR and FFR in 1,593 patients across multiple centers. The authors again concluded that 0.90 is the optimal iFR cutoff for FFR $<0.80$ [C statistic 0.81 (95\% confidence interval, 0.80-0.83); overall accuracy $81.5 \%$ ]. iFR even demonstrated a greater than $90 \%$ accuracy in predicting a positive or negative FFR in a subset of lesions (11).

Proponents of iFR have suggested that lesions with large pressure gradient at hyperemia despite small gradients at rest may be indicative of coronary circulation with well preserved coronary flow reserve or a large contribution of collateral branches. Several ongoing clinical studies will shed further light on the role of iFR and FFR and other functional measures of coronary blood flow $(12,13)$.

\section{FFR and randomized clinical trials}

Prior to FFR being commonly utilized in clinical practice, patients undergoing coronary angiography without prior documented ischemia on noninvasive testing, could undergo coronary revascularization of a lesion without being certain of a future clinical benefit. The publication of three randomized clinical trials in patients with stable CAD has defined the benefit of FFR in interventional practice: (I) DEFER (Fractional Flow Reserve to Determine the Appropriateness of Angioplasty in Moderate Coronary Stenosis) (14); (II) FAME [Fractional Flow Reserve versus Angiography for Guiding percutaneous coronary intervention (PCI) in Patients with Multivessel Coronary Artery Disease] (15); and (III) FAME 2 study (16). Together, these trials have formed the framework behind the evidence for current societal recommendations for FFR-guided coronary revascularization (17).

In the DEFER study, 325 patients with single vessel, stable CAD, without prior documented ischemia on noninvasive testing were examined (14). Patients were randomized to either undergoing percutaneous transluminal coronary angioplasty (PTCA) or deferral of PTCA prior to catheterization, but were ultimately analyzed based on three groups: (I) the defer group (patients randomized to deferral of PTCA, where PTCA was actually deferred based on FFR $\geq 0.75$ ); (II) the perform group (patients randomized to performance of PTCA, where PTCA was actually performed based on FFR $\geq 0.75$ ); and (III) the reference group (where PTCA was performed based on FFR <0.75). There were similar rates of event-free survival between groups, with a significantly lower rate of event-free survival in the reference group compared to the deferral group ( $78 \%$ versus $89 \%$ at 24 months, $\mathrm{P}=0.03$ ). The investigators concluded that in those with CAD and without prior evidence of ischemia, PTCA may be safely deferred in those with stenoses with FFR values $\geq 0.75$.

Following the results of the DEFER trial, the FAME study investigated the use of FFR in patients with stable, multivessel CAD (15). The investigators enrolled 1,005 patients with stenoses of at least $50 \%$. Based on angiographic appearance, these lesions were thought to require percutaneous revascularization. They randomized patients to PCI guided by angiography alone or PCI guided by FFR measurements (FFR $\leq 0.80$ ) in addition to angiography. With respect to the primary endpoint of major adverse cardiac events, the FFR-guided group had a significantly lower rate of events compared to the angiography-guided group $(13.2 \%$ vs. $18.3 \%$, relative risk $0.72, \mathrm{P}=0.02)$. The trial suggested that FFR-guided approach could improve outcomes in patients with stable, multivessel CAD undergoing PCI with the added benefit of fewer stent deployments. The limitations of the FAME study included a lack of a medically treated control group and the inclusion of myocardial infarction as an endpoint, which may have been driven by a high number of post-PCI events in the angiography-guided group.

The FAME 2 trial sought to investigate the use of FFRguided PCI in addition to optimal medical therapy (OMT) compared to OMT alone in patients with stable single vessel or multivessel CAD (16). After 1,220 patients (888 who underwent randomization and 332 enrolled in the registry) were enrolled, further recruitment was stopped because of a significant between-group difference in primary end-point events. In total, 888 patients with at least one FFR positive stenosis (FFR $\leq 0.80$ ) were randomized to either FFR-guided PCI with OMT or OMT alone. The primary composite endpoint of death, myocardial infarction or urgent revascularization at 12 months, the FFR-guided 
PCI group had a significantly lower rate of events $(4.3 \%$ vs. $12.7 \%$, hazard ratio $0.32, \mathrm{P}<0.001)$. This primary endpoint was largely driven by reduction in the need for urgent revascularization, as there was no significant difference in mortality or rate of MI between two groups. At 24 months follow-up, the FFR-guided PCI group continued to demonstrate a lower rate of the primary endpoint $(8.1 \%$ vs. $19.5 \%$, hazard ratio $0.39, \mathrm{P}<0.001)(18)$. This trial suggested that FFR-guided PCI plus OMT strategy led to less urgent revascularization in patients with stable CAD and functionally significant stenosis as compared to OMT alone.

Although the results of FAME 2 trial are encouraging, there are several caveats that should be considered in their interpretation (16). The use of coronary pressures as a surrogate for blood flow can lead to discordance between a normal FFR value and an abnormal coronary flow reserve in some lesions. A meta-analysis examining the prognostic value of FFR revealed a continuous relationship between FFR values and the risk of future adverse events, where lower FFR values confer higher risk, leading to greater benefit with revascularization (19). These findings concurred with subgroup analyses of the FAME 2 population, which revealed that although most patients with FFR significant lesions benefited from PCI, patients with FFR values of $<0.65$ derived the most benefit, as they likely represented the highest risk cohort (20). Importantly, although the initial validation study of FFR against exercise stress electrocardiography used a cutoff value of 0.66 to discriminate for inducible ischemia, subsequent studies have used a higher FFR threshold. Recent investigations comparing FFR to stress echocardiography, nuclear perfusion imaging (21) and stress MRI (22) have all utilized an FFR cutoff range of $0.75-0.80$, which maximizes specificity without sacrificing sensitivity. This is reflected in current societal guidelines, which recommend revascularization for FFR values $<0.80$.

\section{FFR and coronary artery bypass grafting (CABG)}

It is estimated that $>300,000$ CABG surgeries are performed annually in the US at an estimated cost of 10 billion dollars. Since bypass grafts may degenerate and/or close with time, and because native CAD tends to progress in bypassed stretches of coronary anatomy, patients with prior CABG frequently develop recurrent symptoms (23). The operative mortality and morbidity is higher in CABG reoperations than in those undergoing initial surgery and more patients with prior $\mathrm{CABG}$ are presenting for PCI in recent years. At the same time, PCI of bypass grafts is associated with higher rates of both, acute atheroembolic events and longterm adverse events, when compared with PCI of native vessels. The AWESOME (Angina With Extremely Serious Operative Mortality Evaluation) trial collected both randomized, as well as patient and physician-preference registry data, in patients with myocardial ischemia that is refractory to medical therapy with least one of five high-risk factors. This cohort included a subgroup of patients with prior CABG (20). The CABG and PCI 3-year survival rates were not significantly different $[73 \%$ and $76 \%$ respectively for 142 randomized patients $(\mathrm{P}=\mathrm{ns})]$. In the physiciandirected registry, 155 patients were assigned to redo-CABG and 357 to PCI (207 treated medically); 3-year survivals were $71 \%$ and $77 \%$ respectively $(\mathrm{P}=\mathrm{ns})$. In the patientchoice registry, 32 patients chose redo-CABG and 74 chose PCI (13 received medical therapy); 3-year survivals were $65 \%$ and $86 \%$ respectively (log-rank $\mathrm{P}=0.01$ ). These data suggested PCI to be preferable to CABG for many postCABG patients. However, the study lacked clarity given the selection bias within the physician and patient-preference groups and spoke to the need for more randomized data in CABG patients needing repeat revascularization.

Non-invasive functional studies of patients ultimately requiring $\mathrm{CABG}$ surgery [i.e., those with left main (LM) or multi-vessel disease] have lower diagnostic accuracy. Data suggest that $<50 \%$ of patients that were thought to have multivessel disease (MVD) causing ischemia actually had functionally significant MVD (24). Patients with MVD or LM disease on coronary angiography are commonly referred for CABG surgery. Thus, it would be expected that some bypasses be grafted to native vessels without functionally significant lesions (25). Bypass grafting of moderately diseased native vessels may not be effective, and could even be harmful. There are some data that suggest an accelerated atherosclerosis in native vessels, particularly those bypassed with the venous grafts, although other data shows no effect on mortality (26). The arterial grafts have the ability to auto-regulate size and blood flow according to oxygen demand of the perfused myocardium so that the arterial grafts attached to vessels with moderate, functionally non-significant native disease may atrophy or occlude (23). Botman et al. have shown an important relationship between functionally significant coronary stenosis and CABG graft patency at 1 year, where FFR was used to determine a coronary lesion's hemodynamic significance (27). In a prospective study, FFR was 
assessed in stenotic vessels in 164 patients getting surgical revascularization. FFR was measured in all lesions to be grafted, and performing surgeons were blinded to all FFR results. One year after CABG, coronary angiography was performed to evaluate the bypass graft patency rates. After 1 year, angiography revealed $8.9 \%$ of the bypass grafts on functionally significant lesions were occluded, and $21.4 \%$ of the bypass grafts on functionally non-significant lesions were occluded. However, patients with patent or occluded bypass grafts on functionally insignificant lesions did not experience an excess of angina or repeat interventions, and so the long-term clinical implications of these events are not well delineated.

The relationship between FFR and surgical revascularization has not been well studied or understood. The recommendations for FFR are based on large randomized clinical trials data, where patients with typical indications for CABG were excluded. Nam et al. have published a sub-analysis of the FAME trial and have investigated whether an FFR-guided SYNTAX (SYNergy between percutaneous coronary intervention with TAXus and cardiac surgery) score, called "functional SYNTAX score" (FSS), would more accurately predict outcomes than the original SYNTAX score (SS) in patients with MVD undergoing PCI. The FAME study prospectively collected the SS in 497 patients, and so Nam and colleagues developed the FSS in these patients by only counting ischemia-producing lesions (FFR $\leq 0.80$ ). Such FSS analysis has subsequently shifted $32 \%$ of patients into a lower risk group than they were originally assigned (28). Major adverse cardiovascular events (MACE) occurred in $9.0 \%, 11.3 \%$, and $26.7 \%$ of patients in the low-, medium-, and high-FSS groups, respectively $(\mathrm{P}<0.001)$. The FSS was an independent predictor of 1-year MACE, and the adjusted FSS score had a better predictive accuracy for MACE than the original syntax score (28). This study suggested that FFR-guided revascularization could potentially decrease the number of 'higher-risk patients' and better discriminate risk for adverse events in patients with MVD undergoing PCI.

Toth et al. performed a retrospective analysis of patients with intermediate stenosis that were referred for CABG (29). They included 429 patients in the angiography-guided group and 198 patients in the FFR-guided group. Although the rate of MVD was similar between the two groups with angiography alone, there were significantly fewer patients characterized as having MVD after FFR measurements in the FFR-guided group. Additionally, the FFR-guided group was associated with fewer grafts, fewer anastomoses, and a lower rate of on-pump surgery. At 3 years, there were no statistically significant differences in MACE between the angiography-guided and FFR-guided groups (12\% vs. $11 \%$; hazard ratio $1.03, \mathrm{P}=\mathrm{ns})$. However, there was a significantly lower rate of angina in the FFR-guided group compared with the angiography-guided group (Canadian Cardiovascular Society class II-IV, 31\% vs. 47\%; $\mathrm{P}<0.001$ ), which is remarkable given that this group underwent a more limited revascularization strategy. Moreover, the study demonstrated that the rate of graft occlusion was 4 times lower in the FFR-guided group compared with the angiography-guided group.

In a 2004 study, Botman et al. aimed to compare the long-term outcomes of patients who underwent PCIof only hemodynamically significant lesions $(\mathrm{FFR}<0.75$ ) to patients with MVD who underwent CABG of all stenoses (30). If the FFR was $<0.75$ in 3 vessels or in 2 vessels including the proximal left anterior descending artery, CABG was performed (CABG group). If only 1 or 2 vessels were physiologically significant (not including the proximal LAD), PCI of those lesions was performed (PCI group). Of the 150 patients with MVD referred for CABG, 87 fulfilled the criteria for CABG and 63 for PCI. At 2-year follow-up, no differences were seen in MACE, including repeat revascularization (event-free survival 74\% in the CABG group and $72 \%$ in the PCI group). A similar number of patients were free from angina $(84 \%$ in the CABG group and $82 \%$ in the PCI group) (30). This study suggested that in patients with MVD, using FFR to identify hemodynamically significant lesions amenable to $\mathrm{PCI}$ yields a similar favorable outcome as CABG in those with three or more culprit lesions despite a similar angiographic extent of disease.

\section{Limitations of FFR}

\section{Diffuse disease, sequential lesions and bifurcation lesions}

There are several anatomical scenarios where measuring FFR can be technically challenging (e.g., diffuse disease, serial lesions and bifurcation lesions) (4). In patients with diffuse disease or serial lesions, there is a progressive decrease in coronary pressure and blood flow. Although a simple FFR measurement can assess the summed hemodynamic significance of tandem lesions, it may provide inaccurate information for individual stenoses. In patients with diffuse disease, there is a progressive decrease in coronary pressure and flow. The only way to demonstrate the hemodynamic impact of multiple lesions within a 
diffusely diseased vessel is to perform a careful pull-back maneuver of the pressure sensor under steady-state maximal hyperemia. Likewise, when several isolated, but sequential stenoses are present in the same artery, each lesion will influence hyperemic blood. After the most severe stenosis has been treated, pull-back through the residual lesions can be performed to decide whether additional intervention is required. Bifurcation lesions present a particular challenge for FFR measurements. Recent studies by Koo et al. demonstrated that after the main branch is stented, side branch "pinching" can be grossly overestimated by angiography, when confirmed by FFR measurements. The investigators suggest an approach in bifurcation lesions of stenting the main branch and kissing balloon dilation afterward only if FFR of the side branch is $<0.75$, as the angiographic evaluation does not reliably predict the functional significance of a jailed SB stenosis. Using an FFR-guided side branch interventional approach led to no significant difference in 9-month cardiac event rates ( $4.6 \%$ vs. $3.7 \%, \mathrm{P}=0.7$ ) when compared to angiographyguided approach (31). Additionally, Koo and colleagues showed that using a relatively small balloon for side branch dilatation results in significant improvement of functional status of side-branch lesions (31).

\section{LM disease}

Estimating hemodynamic significance of LM stenosis with FFR remains technically challenging. As compared to other coronary arteries, the LM artery is shorter (with an average length of $\sim 10 \mathrm{~mm}$ ) (32) and selective guide engagement is frequently needed to advance the catheter into the coronary system. Operator expertise is required in order to correctly choose and engage the guide catheter, and maintain the guide catheter position in the LM artery. FFR could be misleading in the setting of pressure damping and limited hyperemic flow, thus overestimating the true FFR value. The guide catheter should not have side holes, as the catheter pressure readings may not accurately reflect proximal coronary pressures (5). Use of intracoronary adenosine (as opposed to intravenous adenosine) may not achieve maximal hyperemia. Some authors have suggested advancing the pressure wire past the LM, then completely disengaging the guide catheter from the LM so as to adequately 'normalize' the $\mathrm{Pa}$ and $\mathrm{Pd}$ pressures, and sample the entire length of the LM (including the ostium) on pull-back (33).

\section{MVD}

Patients with MVD frequently have extensive collateralization, which can affect the territory of the myocardium that each stenotic vessel supplies. As the territory supplied by the stenotic vessel decreases, the FFR value increases. Therefore, patients with extensive MVD with multiple collaterals may have FFR values that do not reflect the functional significance of the stenosis in question. However, the FFR measurement in the setting of extensive collaterals may still provide important physiological data regarding the need and benefit of revascularization.

\section{Microvascular disease}

In patients with severe microvascular disease, hyperemia cannot be reliably achieved. The fixed downstream resistance to increased flow in the setting of vasodilators limits flow capacity through the more proximal coronary artery. This resistance artificially elevates pressure measurements distal to an epicardial stenosis, and will result in an increase in measured FFR. Two studies found that FFR has a substantially lower accuracy in patients with moderate to severe microvascular disease than for those with less microvascular disease, thus obscuring the ability of FFR to detect important epicardial disease in these patients $(34,35)$. In such situations, alternate hemodynamic measurements (such as CFR) should be considered (36).

\section{Acute coronary syndromes (ACS)}

In patients with ACS, culprit lesions can affect the downstream vasculature and myocardium through several mechanisms, including a down-regulation of vascular receptors, endothelial impairment, or vasoconstriction via sympathetic activation or vasospasm (37). Such downstream injury decreases that tissue's ability to achieve maximal hyperemia and may also impact non-culprit territories through similar mechanisms. This increased resistance to hyperemia will, by definition, falsely raise the value of measured FFR. Higher FFR may lead to the deferral of culprit-vessel revascularization, which could theoretically be deleterious, as revascularization in the setting of ACS has been shown to improve clinical outcomes in the era prior to routine use of FFR (38). Furthermore, in light of growing evidence that non-culprit lesion revascularization 
can improve outcomes, particularly in ST-elevation ACS, the increase in measured FFR could lead to lower rates of "complete" revascularization (39). Three moderatesized, randomized clinical trials examining the optimal management of non-culprit lesions in ACS found that $38-56 \%$ of non-culprit lesions that have at least $50 \%$ diameter stenosis by angiography have an FFR $>0.8$. Patients randomized to FFR-guided revascularization had lower absolute rates of revascularization, but overall similar clinical outcomes (40-42). Further studies are necessary to examine the utilization of FFR in this high-risk cohort.

\section{Heart failure}

The increased CVP and left ventricular end-diastolic pressure (LVEDP) in patients with heart failure may affect the hemodynamic assumptions made in the calculation of FFR. In right-sided heart failure, elevated CVP decreases coronary flow by decreasing the pressure gradient across the coronary vasculature and thus may affect the FFR measurement leading to underestimation of true FFR. However, recent data suggest that even extreme right atrial pressures would only marginally affect the FFR values (43). In left-sided heart failure, an elevated LVEDP directly impedes myocardial perfusion by creating functional microvascular resistance (44). Experimentally, Leonardi et al. demonstrated that there was a positive association between LVEDP and FFR (45). Specifically, they found that for every $1 \mathrm{mmHg}$ of increase in LVEDP, there was an increase in FFR of 0.008 by measuring FFR and LVEDP simultaneously in the presence and absence of nitroprusside. In those patients where baseline FFR was $<0.8$, FFR increased by 0.01 for every $1 \mathrm{mmHg}$ rise in LVEDP.

\section{Valvular disease}

Concomitant valvular disease can affect the FFR measurements. For instance, patients with aortic stenosis may have elevated LVEDP and left ventricular hypertrophy. These factors increase microvascular resistance in a manner analogous to patients with left-sided heart failure, and will raise the measured FFR. Clinically, this may lead to more coronary lesions being "downgraded" in number or severity. Di Gioia et al. demonstrated that use of FFR in patients with moderate to severe aortic stenosis could lead to significant downgrading of the number of coronary lesions compared to angiography alone in matched controls, which lead to increased use of PCI and lower rates of CABG (46).
Interestingly, there was no difference in incidence of major adverse cardiac events between the two groups at a median 56 months follow up. More data are needed to determine the impact and validity of FFR on clinically relevant outcomes in patients with concomitant valvular disease.

\section{FFR in post-CABG patients}

PCI of bypass grafts is associated with higher rates of MACE as compared to PCI of native vessels mainly due to higher periprocedural myocardial infarction and repeat revascularization rates. Because of the nature of friable plaque in grafts, there is a higher risk of embolization into downstream native coronary circulation. However, PCI of bypass grafts is frequently preferred to reoperation, and drug-eluting stents use in grafts has been associated with improved outcomes and fewer repeat interventions $(23,47)$.

Di Serafino et al. evaluated the long-term clinical outcomes of prior CABG patients undergoing FFR-guided PCI of intermediate graft lesions (both arterial and venous) versus angiography-guided PCI approach (48). Patients were included with stable and unstable angina with at least 1 intermediate stenosis and $96 \%$ had clinical follow-up for a median of 3.8 years. There were no significant differences in patient characteristics, including total number of bypass grafts (65 in FFR-guided patients and 158 in angiographyguided patients). In the FFR-guided group, 35\% underwent PCI compared to $57 \%$ in the angiography-guided group. FFR-guided PCI had a significantly lower MACE and cerebrovascular events at 4-year follow-up, even after propensity score adjustment. In the FFR-guided group, PCI was more frequently performed on arterial grafts, and the overall clinical benefit was more significant in arterial grafts. In the angiography-guided group, PCI was performed in more venous graft cases, although the distal embolic protection devices were used in $<10 \%$ cases (48), which may have accounted for the higher event rate.

The main challenge of using FFR in post-CABG patients is presence of competing flow and pressure from native vessels and grafts, as well as, the presence of collaterals. Measurements of FFR across bypass graft stenoses are different, depending on whether the native coronary artery is occluded or patent. If the native vessel is occluded, the procedure and interpretation of FFR results are the same as standard FFR protocol. But if the native vessel is patent, and competitive flow is present, the pressure wire sensor should be placed distally to the anastomosis to account for the disease burden of both graft and native artery on the 
perfusion of the downstream myocardial territory. If FFR value is $<0.80$ when competitive flow is present, there is inadequate perfusion of that territory from the native artery and bypass graft combined. With jump grafts, data suggest that FFR measurement can still be reliable, although they are vulnerable to the pitfalls of using FFR for serial stenoses and bifurcation lesions (23).

Another consideration in evaluating post-CABG patients with FFR is that resistance to flow is different in arterial and venous grafts. Saphenous vein grafts are larger than native coronary arteries and vein graft anastomoses may cause a pressure drop. Arterial grafts produce nitric oxide, which may affect pressure differential as compared to vein grafts. The left internal mammary artery's (LIMA) resistance is higher than that of the right internal mammary artery (RIMA) or vein graft, and so there is often a lower FFR and higher pressure drop in the LIMA. Differences in resistance and FFR may also be related to myocardial territory size; for example, the LIMA is usually implanted to the LAD, which generally supplies the largest myocardial territory. Furthermore, configurations of some grafts (kinking, large curvatures) could affect pressure wave transmission. Lastly, differences in length and lumen size may lead to inherent differences in resistances.

There are currently ongoing randomized, blinded control trials that are investigating patency rates and MACE in FFR-guided revascularization. For example, the GRAFFITI trial is examining 1-year patency rates in patients with MVD after angiography-guided versus FFRguided surgical strategies. By implementing FFR-guided CABG strategy, coronary segments with functionally significant disease could more easily be offered full functional arterial revascularization by implanting the best available arterial vascular conduits. FFR-guided decision making regarding revascularization may also lead to more frequent hybrid revascularizations, particularly in patients with borderline functionally significant stenoses of coronary vessels (23).

\section{Conclusions}

FFR has become an important tool to guide the need for revascularization and in determining the best revascularization strategy in MVD. It is important to understand the principles guiding FFR and its limitations. Several randomized controlled trials have demonstrated the potential benefit of FFR-guided strategy with regard to clinical outcomes or further revascularization in stable
CAD. The available data suggest that FFR may be a useful tool in managing patients before and after surgical revascularization. The strategy and outcomes of functionally complete revascularization need to be further examined in future clinical trials.

\section{Acknowledgements}

None.

\section{Footnote}

Conflicts of Interest: The authors have no conflicts of interest to declare.

\section{References}

1. Algranati D, Kassab GS, Lanir Y. Why is the subendocardium more vulnerable to ischemia? A new paradigm. Am J Physiol Heart Circ Physiol 2011;300:H1090-100.

2. Kern MJ. Coronary physiology revisited: practical insights from the cardiac catheterization laboratory. Circulation 2000;101:1344-51.

3. Pijls NH, van Son JA, Kirkeeide RL, et al. Experimental basis of determining maximum coronary, myocardial, and collateral blood flow by pressure measurements for assessing functional stenosis severity before and after percutaneous transluminal coronary angioplasty. Circulation 1993;87:1354-67.

4. Pijls NH, De Bruyne B, Peels K, et al. Measurement of fractional flow reserve to assess the functional severity of coronary-artery stenoses. N Engl J Med 1996;334:1703-8.

5. Toth GG, Johnson NP, Jeremias A, et al. Standardization of Fractional Flow Reserve Measurements. J Am Coll Cardiol 2016;68:742-53.

6. Pijls NH, Kern MJ, Yock PG, et al. Practice and potential pitfalls of coronary pressure measurement. Catheter Cardiovasc Interv 2000;49:1-16.

7. Nijjer SS, Sen S, Petraco R, et al. Advances in coronary physiology. Circulation 2015;79:1172-84.

8. Nijjer SS, Sen S, Petraco R, et al. Improvement in coronary haemodynamics after percutaneous coronary intervention: assessment using instantaneous wave-free ratio. Heart 2013;99:1740-8.

9. Sen S, Escaned J, Malik IS, et al. Development and validation of a new adenosine-independent index of stenosis severity from coronary wave-intensity analysis: 
results of the ADVISE (ADenosine Vasodilator

Independent Stenosis Evaluation) study. J Am Coll Cardiol 2012;59:1392-402.

10. Petraco R, Al-Lamee R, Gotberg M, et al. Real-time use of instantaneous wave-free ratio: results of the ADVISE in-practice: an international, multicenter evaluation of instantaneous wave-free ratio in clinical practice. Am Heart J 2014;168:739-48.

11. Jeremias A, Maehara A, Genereux P, et al. Multicenter core laboratory comparison of the instantaneous wave-free ratio and resting $\mathrm{Pd} / \mathrm{Pa}$ with fractional flow reserve: the RESOLVE study. J Am Coll Cardiol 2014;63:1253-61.

12. Petraco R, van de Hoef TP, Nijjer S, et al. Baseline instantaneous wave-free ratio as a pressure-only estimation of underlying coronary flow reserve: results of the JUSTIFY-CFR Study (Joined Coronary Pressure and Flow Analysis to Determine Diagnostic Characteristics of Basal and Hyperemic Indices of Functional Lesion Severity-Coronary Flow Reserve). Circ Cardiovasc Interv 2014;7:492-502.

13. Sen S, Asrress KN, Nijjer S, et al. Diagnostic classification of the instantaneous wave-free ratio is equivalent to fractional flow reserve and is not improved with adenosine administration. Results of CLARIFY (Classification Accuracy of Pressure-Only Ratios Against Indices Using Flow Study). J Am Coll Cardiol 2013;61:1409-20.

14. Bech GJ, De Bruyne B, Pijls NH, et al. Fractional flow reserve to determine the appropriateness of angioplasty in moderate coronary stenosis: a randomized trial. Circulation 2001;103:2928-34.

15. Tonino PA, De Bruyne B, Pijls NH, et al. Fractional flow reserve versus angiography for guiding percutaneous coronary intervention. N Engl J Med 2009;360:213-24.

16. De Bruyne B, Pijls NH, Kalesan B, et al. Fractional flow reserve-guided PCI versus medical therapy in stable coronary disease. N Engl J Med 2012;367:991-1001.

17. Levine GN, Bates ER, Blankenship JC, et al. 2011 ACCF/AHA/SCAI Guideline for Percutaneous Coronary Intervention: a report of the American College of Cardiology Foundation/American Heart Association Task Force on Practice Guidelines and the Society for Cardiovascular Angiography and Interventions. Circulation 2011;124:e574-651.

18. De Bruyne B, Fearon WF, Pijls NH, et al. Fractional flow reserve-guided PCI for stable coronary artery disease. N Engl J Med 2014;371:1208-17.

19. Johnson NP, Toth GG, Lai D, et al. Prognostic value of fractional flow reserve: linking physiologic severity to clinical outcomes. J Am Coll Cardiol 2014;64:1641-54.

20. Elguindy AM, Bonow RO. FAME 2 - The best initial strategy for patients with stable coronary artery disease: Do we have an answer at last? Glob Cardiol Sci Pract 2012;2012:15-7.

21. Pattanshetty DJ, Bhat PK, Gandhi S, et al. Comparing stress testing and fractional flow reserve to evaluate presence, location and extent of ischemia in coronary artery disease. Indian Heart J 2015;67:50-5.

22. Rieber J, Huber A, Erhard I, et al. Cardiac magnetic resonance perfusion imaging for the functional assessment of coronary artery disease: a comparison with coronary angiography and fractional flow reserve. Eur Heart J 2006;27:1465-71.

23. Pellicano M, De Bruyne B, Toth GG, et al. Fractional flow reserve to guide and to assess coronary artery bypass grafting. Eur Heart J 2016. [Epub ahead of print].

24. Tonino PA, Fearon WF, De Bruyne B, et al. Angiographic versus functional severity of coronary artery stenoses in the FAME study fractional flow reserve versus angiography in multivessel evaluation. J Am Coll Cardiol 2010;5 5:2816-21.

25. Cosgrove DM, Loop FD, Saunders CL, et al. Should coronary arteries with less than fifty percent stenosis be bypassed? J Thorac Cardiovasc Surg 1981;82:520-30.

26. Sabik JF 3rd, Olivares G, Raza S, et al. Does grafting coronary arteries with only moderate stenosis affect longterm mortality? J Thorac Cardiovasc Surg 2016;151:806-11

27. Botman CJ, Schonberger J, Koolen S, et al. Does stenosis severity of native vessels influence bypass graft patency? A prospective fractional flow reserve-guided study. Ann Thorac Surg 2007;83:2093-7.

28. Nam CW, Mangiacapra F, Entjes R, et al. Functional SYNTAX score for risk assessment in multivessel coronary artery disease. J Am Coll Cardiol 2011;58:1211-8.

29. Toth G, De Bruyne B, Casselman F, et al. Fractional flow reserve-guided versus angiography-guided coronary artery bypass graft surgery. Circulation 2013;128:1405-11.

30. Botman KJ, Pijls NH, Bech JW, et al. Percutaneous coronary intervention or bypass surgery in multivessel disease? A tailored approach based on coronary pressure measurement. Catheter Cardiovasc Interv 2004;63:184-91.

31. Koo BK, Park KW, Kang HJ, et al. Physiological evaluation of the provisional side-branch intervention strategy for bifurcation lesions using fractional flow reserve. Eur Heart J 2008;29:726-32.

32. Abedin Z, Goldberg J. Origin and length of left main coronary artery: its relation to height, weight, sex, age, pattern of coronary distribution, and presence or absence 
of coronary artery disease. Cathet Cardiovasc Diagn 1978;4:335-40.

33. Kern MJ. MY APPROACH to the patient diagnosed with significant left main disease: Use of FFR, IVUS, and OCT. Trends Cardiovasc Med 2016;26:660-1.

34. van de Hoef TP, Nolte F, EchavarrIa-Pinto M, et al. Impact of hyperaemic microvascular resistance on fractional flow reserve measurements in patients with stable coronary artery disease: insights from combined stenosis and microvascular resistance assessment. Heart 2014;100:951-9.

35. Meuwissen M, Chamuleau SA, Siebes M, et al. Role of variability in microvascular resistance on fractional flow reserve and coronary blood flow velocity reserve in intermediate coronary lesions. Circulation 2001;103:184-7.

36. Johnson NP, Kirkeeide RL, Gould KL. Is discordance of coronary flow reserve and fractional flow reserve due to methodology or clinically relevant coronary pathophysiology? JACC Cardiovasc Imaging 2012;5:193-202.

37. Johnson NP, Gould KL, Di Carli MF, et al. Invasive FFR and Noninvasive CFR in the Evaluation of Ischemia: What Is the Future? J Am Coll Cardiol 2016;67:2772-88.

38. Fox KA, Clayton TC, Damman P, et al. Long-term outcome of a routine versus selective invasive strategy in patients with non-ST-segment elevation acute coronary syndrome a meta-analysis of individual patient data. J Am Coll Cardiol 2010;55:2435-45.

39. Engstrom T, Kelbaek H, Helqvist S, et al. Complete revascularisation versus treatment of the culprit lesion only in patients with ST-segment elevation myocardial infarction and multivessel disease (DANAMI-3PRIMULTI): an open-label, randomised controlled trial. Lancet 2015;386:665-71.

40. Layland J, Oldroyd KG, Curzen N, et al. Fractional flow reserve vs. angiography in guiding management to optimize outcomes in non-ST-segment elevation myocardial infarction: the British Heart Foundation

Cite this article as: Shah T, Geleris JD, Zhong M, Swaminathan RV, Kim LK, Feldman DN. Fractional flow reserve to guide surgical coronary revascularization. J Thorac Dis 2017;9(Suppl 4):S317-S326. doi: 10.21037/jtd.2017.03.55
FAMOUS-NSTEMI randomized trial. Eur Heart J 2015;36:100-11.

41. Sels JW, Tonino PA, Siebert U, et al. Fractional flow reserve in unstable angina and non-ST-segment elevation myocardial infarction experience from the FAME (Fractional flow reserve versus Angiography for Multivessel Evaluation) study. JACC Cardiovasc Interv 2011;4:1183-9.

42. Wald DS, Morris JK, Wald NJ, et al. Randomized trial of preventive angioplasty in myocardial infarction. N Engl J Med 2013;369:1115-23.

43. Toth GG, De Bruyne B, Rusinaru D, et al. Impact of Right Atrial Pressure on Fractional Flow Reserve Measurements: Comparison of Fractional Flow Reserve and Myocardial Fractional Flow Reserve in 1,600 Coronary Stenoses. JACC Cardiovasc Interv 2016;9:453-9.

44. Van Herck PL, Carlier SG, Claeys MJ, et al. Coronary microvascular dysfunction after myocardial infarction: increased coronary zero flow pressure both in the infarcted and in the remote myocardium is mainly related to left ventricular filling pressure. Heart 2007;93:1231-7.

45. Leonardi RA, Townsend JC, Patel CA, et al. Left ventricular end-diastolic pressure affects measurement of fractional flow reserve. Cardiovasc Revasc Med 2013;14:218-22.

46. Di Gioia G, Pellicano M, Toth GG, et al. Fractional Flow Reserve-Guided Revascularization in Patients With Aortic Stenosis. Am J Cardiol 2016;117:1511-5.

47. Aggarwal V, Stanislawski MA, Maddox TM, et al. Safety and effectiveness of drug-eluting versus bare-metal stents in saphenous vein bypass graft percutaneous coronary interventions: insights from the Veterans Affairs CART program. J Am Coll Cardiol 2014;64:1825-36.

48. Di Serafino L, De Bruyne B, Mangiacapra F, et al. Longterm clinical outcome after fractional flow reserve- versus angio-guided percutaneous coronary intervention in patients with intermediate stenosis of coronary artery bypass grafts. Am Heart J 2013;166:110-8. 